\title{
Transiciones sociales y los retos de la enseñanza online
}

\section{Social transitions and the challenges of online teaching}

DOI: $10.46932 / \mathrm{sfjdv2n5-111}$

Received in: Oct 1st, 2021

Accepted in: Dec 30th, 2021

\section{Barbara Eliana Coaguila-Mitta}

Doctor en Ciencias de la Educación, Maestro en Educación: Docencia y Gestión Educativa, Licenciada en Trabajo Social

Universidad Nacional de San Agustín de Arequipa

Arequipa, Perú

E-mail: bcoaguilam@unsa.edu.pe

\section{Karen Haydee Pastor-Xespe}

Maestro en Gerencia Social y de Recursos Humanos, Licenciada en Trabajo Social

Universidad Nacional de San Agustín de Arequipa

Arequipa, Perú

E-mail: kpastorx@unsa.edu.pe

\section{Rosa María Vela-Aquize}

Maestro en Gerencia Social y de Recursos Humanos, Licenciada en Trabajo Social

Universidad Nacional de San Agustín de Arequipa

Arequipa, Perú

E-mail: rvelaa@unsa.edu.pe

\section{Roberto Cervantes-Rivera}

Maestro en Gerencia Social y de Recursos Humanos, Licenciada en Historia

Universidad Nacional de San Agustín de Arequipa

Arequipa, Perú

E-mail: rcervantesr@unsa.edu.pe

\section{Deyvin Herlin Cabana-Mamani}

Maestro en gerencia social y de recursos humanos, Licenciado en Turismo y Hotelería, Bachiller en Derecho.

Universidad Nacional de San Agustín de Arequipa

Arequipa, Perú

E-mail: dcabanama@unsa.edu.pe

\section{RESUMEN}

El presente trabajo aborda una revisión bibliográfica sobre los aspectos más relevantes que han motivado los cambios o transiciones sociales en los últimos tiempos. Se revisan diversos documentos académicos con el fin de conocer si estos cambios sociales han afectado de una u otra forma las metodologías educativas, y más aun la enseñanza online, que ha representado un importante cambio en la época reciente. Y, además, si esta metodología online puede garantizar resultados similares o mejores a la educación presencial. Los resultados muestran que la educación tradicional es la que continúa vigente en los tiempos 
actuales, y es la que ha estado presente durante años, que la sociedad ha sufrido importantes cambios y evoluciones, pero la educación es en esencia la misma.

Palabras clave: transiciones sociales, metodologías educativas, enseñanza online.

\begin{abstract}
The present work addresses a bibliographic review on the most relevant aspects that have motivated social changes or transitions in recent times. Various academic documents are reviewed in order to know if these social changes have affected educational methodologies in one way or another, and even more so online teaching, which has represented an important change in recent times. And, also, if this online methodology can guarantee similar or better results to face-to-face education. The results show that traditional education is the one that continues in force today, and it is the one that has been present for years, that society has undergone important changes and evolutions, but education is essentially the same.
\end{abstract}

Keywords: social transitions, educational methodologies, online teaching.

\title{
1 INTRODUCCIÓN
}

La sociedad ha venido evolucionando desde siempre, y estos cambios han condicionado la conducta de las personas, sus hábitos y sus preferencias. La forma de vida, las necesidades laborales, y todos los aspectos relacionados con el intercambio social entre las personas, se ha visto afectado por cada época y por cada transición social. Sin embargo, el eje fundamental de estos cambios ha sido la tecnología y sus repercusiones se observan en diversas áreas, pero principalmente en la educación.

Los cambios sociales tienen diversas fuentes, que pueden incluir factores demográficos, culturales, tecnológicos o ideológicos. En todos los casos, los cambios producen un efecto en la conducta social, y pueden ser condicionantes para cambios sucesivos en el individuo, de tal manera que un cambio social puede conducir a cambios familiares, individuales, etc.

La educación tiene sus orígenes en la antigüedad, incluso podríamos suponer que se inicia con las sociedades primitivas, cuando los mayores debían enseñarle a los más pequeños, las técnicas de recolección de alimentos, de caza de animales, de pesca, o de supervivencia para la vida cotidiana (La importancia de las letras, 2010). Cada evento en las comunidades primitivas pudo estar inmerso en un proceso educativo para los jóvenes, desde la trascendencia cultural hasta las implicaciones para la vida y para los demás miembros de la comunidad.

A medida que las formas de vida humana evolucionan, se cambian los procesos educativos, se incorporan nuevas tecnologías y nuevas metodologías, que estarán condicionadas a las tendencias o cambios sociales, que finalmente son los que dan directrices para las adaptaciones educativas.

Algunos autores (Guichot, 2006) afirman que los procesos educativos han ido ocurriendo en concordancia con los comportamientos sociales, con prácticas educativas determinadas por el rol del 
maestro, y asociada con las condiciones profesionales, culturales, políticas que han promovido el orden jerárquico y jurídico de la educación.

En este trabajo se analiza la evolución humana desde una perspectiva social, y se evalúan las metodologías educativas que han estado en sintonía con esa transición social, y que han tenido que adaptarse a las características sociales que presenta cada época. Sin embargo, se vislumbran algunos criterios que requieren atención.

La sociedad, es sin duda, un espacio para el intercambio de las personas, donde confluyen creencias y culturas, costumbres, tradiciones, factores económicos, aspectos políticos, y formas de vida variadas. Todos estos, son determinantes para el estudio aquí planteado, que además de considerar los cambios sociales, evalúa su impacto en las transiciones académicas y educativas de esa sociedad fluctuante.

\section{DESARROLLO}

La sociedad se define como un conjunto de personas que coordinan ciertas normas para cumplir algunos fines de la vida (Westreicher, Sociedad, 2020), es decir, que existe un orden común, y por ende, se establecen tradiciones, costumbres e incluso la moneda y otros aspectos económicos. La sociedad actual se centra en la producción y el desarrollo, y se formulan ideologías como la de querer algo es hacerlo posible (Scafatti, s.f.).

\subsection{ORIGEN DE LAS SOCIEDADES HUMANAS}

El inicio de la agricultura fue determinante para dar origen a las sociedades modernas, ya que las necesidades de sedentarismo de los pobladores, para realizar los cultivos y la siembra, los obligó a establecer normas de convivencia social, y a su vez, establecer una organización económica de intercambio.

Las diferentes variantes de organización social empezaron a formarse según las características de la producción de las mismas, así entonces se forma la sociedad de caza y recolección, la agricultura y pesca y luego la industrial.

Además de esto, se han concebido estructuras sociales a partir de las estructuras políticas, que constituyen la formación de bandas, tribus, estados, jefaturas, entre otras formas de orden. Pero en todos los casos, las sociedades aisladas tienen mayor probabilidad de continuar en el tiempo que aquellas que están en entornos más competitivos, con mayores riesgos de invasión o de solapamiento de espacios.

Algunos autores afirman que las sociedades se pueden diferenciar según el uso y nivel de la tecnología, de la forma de comunicarse y de la forma económica de organizarse (Lenski, 1974). Pero 
existen varias formas de ver las sociedades, algunos autores aseguran que se trata de una creación del hombre y para el hombre, con constantes cambios producidos por el hombre también (Dunfey, 2019).

\subsubsection{Características de la sociedad}

La figura 1 describe los elementos más relevantes de las estructuras sociales, se distinguen algunos aspectos que caracterizan la vida social como:

Las costumbres condicionan los lazos entre las personas que conforman los grupos sociales, logrando un sentido de pertenencia entre los miembros. Adicional a ello, los grupos que comparten historias, pueden fortalecer sus vínculos afectivos.

Las sociedades se organizan en grupos focalizados en sectores o espacios geográficos, que, según sus características, también condicionarán otros aspectos de la sociedad, como la vida económica.

Se establecen normas y formas de vida, con el fin de garantizar una buena convivencia.

Las sociedades evolucionan, sus normas se ajustan a esos cambios.

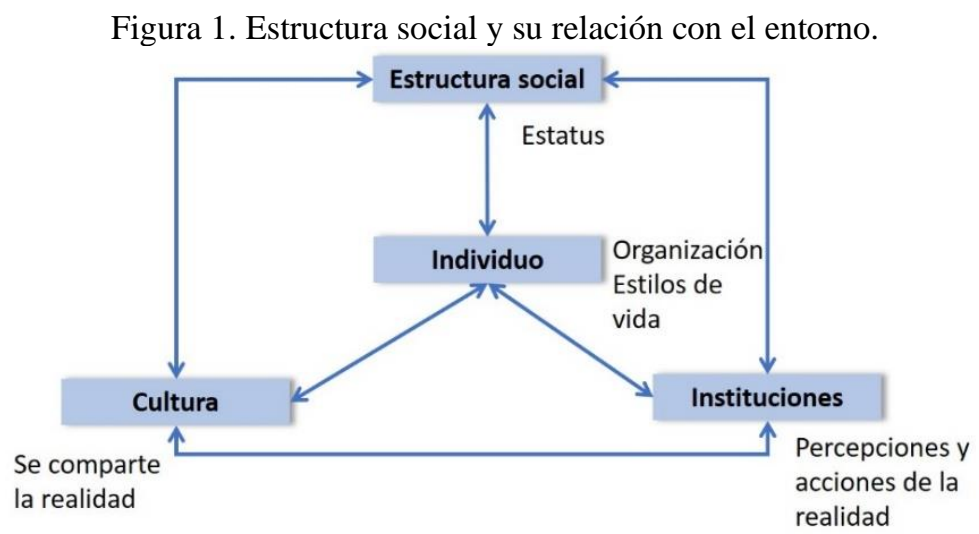

Fuente: (Ssociólogos, 2012)

\subsubsection{Tipos de sociedades}

Como ya se ha mencionado antes, las sociedades se pueden agrupar según el tipo de actividad que realicen y las formas de organizarse (Westreicher, Sociedad, 2020). De tal manera que se pueden distinguir los siguientes tipos de sociedad:

Sociedades primitivas: tratan de los primeros grupos sociales, formados por personas que se dedicaban a la caza, la pesca y la recolección.

Sociedades pastorales: dedicadas principalmente a la ganadería y el cuidado de animales para la producción.

Sociedades hortícolas: dieron paso a la agricultura, se originaron en Asia. Más adelante poblaron importantes sectores de Europa. 
Sociedad agrícola: Este tipo de sociedad fue aun más organizada que las anteriores, consideró un mejor aprovechamiento del agua, y empezaron a usar los animales para la vida económica y las mejoras en los procesos del campo.

Sociedad medieval: nacen las jerarquías sociales, se forman grupos y formas políticas sociales.

Sociedad industrial: Se forman los estados, se desarrollan procesos de ingeniería destacado, se da un cambio importante en la forma laboral y nace la industrialización. Cambian las visiones de las personas por el futuro.

Sociedad post industrial: Se destaca por la formación de grupos de servicios, es una sociedad más avanzada, la industria sufre una transformación más global.

\subsection{HISTORIA DE LA EDUCACIÓN}

En este apartado se considerarán los momentos más destacados de la educación a lo largo de la historia, para ello se evaluarán los criterios descritos en la figura 2.

Figura 2. Evolución y transiciones de la educación.

Educación

Tradicional

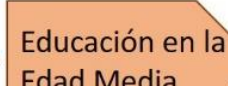

Edad Media

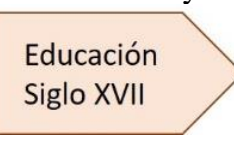

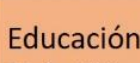

Siglo XIX
Educación Siglo XX
Educación Siglo XXI

Se considera como educación tradicional aquella compuesta por costumbres repetidas, donde se aprecia una continuidad de las ideologías, y prevalece el método de Sócrates (Zambrano, 2018). Los autores principales de la educación tradicional son Platón, Sócrates y Aristóteles. La educación estaba centrada en la formación de la persona para su vida, se consideraba que los procesos de enseñanza podían garantizar una calidad de vida. En Atenas se prohibió la formación de soldados, en cambio se centraban en la cultura, las matemáticas y la escritura. Por otro lado, en Esparta se fundamentaba la educación para soldados. El ideal educativo de Esparta no estaba centrado en la vida plena del individuo, sino en las comunidades de soldados.

En la edad media, el analfabetismo era predominante en la población, y solo sabían escribir y leer aquellas personas de la alta sociedad letrados, del clérigo, de aquellos con estudios especializados. En esta época predominó el estudio del buen uso de la lengua, el lenguaje con buena pronunciación y la poética. Además, se dio prioridad al humanismo. Se establece la educación obligatoria para todas las personas, se establece además la formación del clero y los seminarios religiosos.

Luego surge la didáctica magna, que promueve a la pedagogía como ciencia y centra al alumno como eje esencial de la enseñanza (Zambrano, 2018). Se formulan debates entorno a las formas de la enseñanza, ya que se incorpora el concepto de experimentación para la educación. Con estos cambios, nacen las primeras universidades y se da inicio a la educación superior. 
Más adelante, se democratiza la educación, y se da inicio a la educación publicación obligatoria. Se establecen los centros de enseñanza superior. Se da importancia a la personalidad de los estudiantes y se valora el ambiente y el entorno en la educación. Se universalizan estas características de la educación.

En el siglo XXI la educación se fundamenta en el uso de las tecnologías y las comunicaciones a través de equipos y aparatos electrónicos. Se crean las carreras online y se le da prioridad a la enseñanza virtual, con interacciones globales y una visión de desarrollo de forma inmediata. Se refuerzan las aplicaciones tecnológicas, se crean metodologías para la enseñanza virtual pero no se fundamenta en una visión filosófica.

\subsection{RETOS DE LA ENSEÑANZA ONLINE}

Las nuevas tendencias en educación, conducen a la utilización de herramientas tecnológicas que aporten a la enseñanza, y a su vez la utilización de plataformas virtuales que promuevan la educación a distancia. Con esto, la educación sufre un cambio de esquemas, más que de paradigmas, y estos cambios se enfocan en el uso de múltiples herramientas y metodologías que facilitan el aprendizaje y que aportan de forma práctica a la enseñanza.

Sin embargo, la visión virtual de la enseñanza aun no está definida con claridad para algunos sistemas y se ha convertido la clase virtual en un escenario igual a la educación tradicional, encasillando a los estudiantes a escenarios donde el profesor dicta de forma inequívoca una asignatura y donde los exámenes son supervisados de la misma forma que en la edad media.

Se crean espacios y plataformas de alto rendimiento y grandes herramientas para la comunicación entre el profesor y los estudiantes, sin embargo, se realizan metodologías tradicionales donde el docente imparte de la misma manera el conocimiento y las evaluaciones están limitadas a la supervisión del profesor de forma clásica.

En la figura 3 se observan los métodos tradicionales de enseñanza y los métodos modernos de la educación virtual, puede observarse que es la misma aula y que solo cambian los escenarios. Incluso en la educación virtual el proceso de evaluación consiste en la supervisión docente de los estudiantes, con la condición de que estos enciendan las cámaras y puedan ser monitorizados. Lo que resulta igual a los métodos clásicos.

Figura 3. Educación tradicional, donde el docente muestra sus conocimientos a los estudiantes en un espacio físico, en contraste con la educación virtual, donde el docente muestra sus conocimientos a los estudiantes en un espacio virtual.
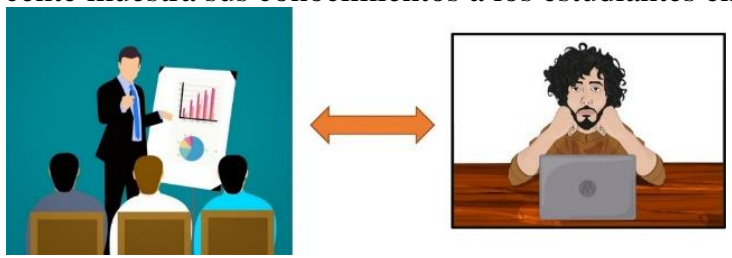

Fuente: (Pixabay, s.f.) 
En función de esto es importante que se evalúen las metodologías de enseñanza y que se evalúen las necesidades de profesionales más adaptados a la visión global de la sociedad actual, de tal manera que sea posible valorar las necesidades profesionales que requiere la industria y en función a esto reformular las formas de enseñar.

El reto de la educación es romper con sus propios esquemas, para que se de apertura a un profesional apto para la nueva visión industrial de los nuevos tiempos. Estos retos deben conjugarse con la formación de educadores con visión global, que puedan adaptar los procesos de enseñanza a una nueva forma, que integre no solo conocimiento sino, además, independencia, individualidad, multidisciplinariedad, y habilidades de investigación y ciencias.

\section{METODOLOGÍA}

El siguiente trabajo ha sido elaborado siguiendo una metodología de revisión bibliográfica, para ello se han revisado un importante número de documentos que reflejan los indicadores primordiales para este este análisis (Calizaya, y otros, 2020). Se evalúan los criterios de inclusión y exclusión señalados en la tabla 1.

Se analizan los elementos que conducen a la enseñanza de las personas, desde su visión social y su impacto en la conducta colectiva del ser humano. Se consideran diferentes épocas y diferentes escenarios de acción de la vida humana y de su evolución en el tiempo, para dar inicio a la enseñanza y a los procesos educativos.

Tabla 1. Criterios de inclusión y exclusión utilizados en las cadenas de búsquedas de la revisión bibliográfica.

\begin{tabular}{|c|l|}
\hline \multicolumn{1}{|c|}{ Criterios de inclusión } & Criterios de exclusión \\
\hline $\begin{array}{l}\text { Palabras clave: educación, enseñanza, } \\
\text { sociedad, evolución social, metodologías de } \\
\text { enseñanza }\end{array}$ & Publicaciones sin validez científica \\
\hline Material académico & Opiniones personales \\
\hline Acceso abierto & Sectorización por países \\
\hline Pertinencia & Visión no globalizada \\
\hline Actualidad & Desactualizado \\
\hline
\end{tabular}

El proceso de revisión estuvo compuesto de cuatro fases fundamentales, descritas en la figura 4.

Figura 4. Fases del proceso de revisión bibliográfica seguido para el estudio
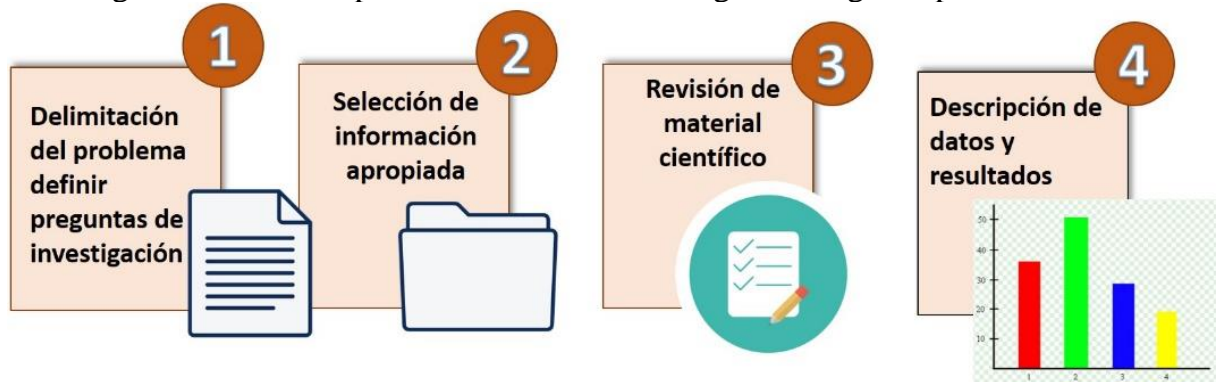


\section{RESULTADOS}

Una vez realizada la revisión documental, se han podido observar ciertos criterios de relevancia que conducen a la generación de los siguientes resultados:

1. La educación tradicional estaba centrada en el estudiante, sin embargo, carecía de otros elementos como la empatía y el respeto a la personalidad, se daba gran importancia a la participación del docente y se consideraban estos como sabios con una única verdad.

2. La educación ha estado colmada de situaciones sociales, que deberían condicionar las metodologías de enseñanza, sin embargo, se ha mantenido un criterio similar de enseñanza a lo largo de la historia. Las aulas continúan siendo espacios cerrados donde una única persona transmite los conocimientos. A pesar de los intentos por lograr una educación diferente, el principio fundamental de la enseñanza sigue siendo el mismo.

3. Las metodologías educativas deben ser adaptativas a su época y deben considerar las implicaciones sociales que ella conduce, además debe focalizar sus esfuerzos en el profesional que se desea para el tiempo y espacio al que este pertenece, con el fin de que la enseñanza sea óptima y eficiente, y que cada vez más los profesionales puedan dar respuesta a las exigencias industriales a las que corresponden.

4. La enseñanza virtual es un reto para las sociedades modernas, y ello obliga a la necesidad de transformación, y hasta aquí pareciera imposible sostener las formas de enseñanza tradicionales, que han prevalecido de forma ininterrumpida a lo largo de los años, pero ahora, con las necesidades sociales envueltas en un uso desmedido de la tecnología, con las nuevas exigencias industriales enfocadas al aprovechamiento tecnológico, los retos de la educación deben ser aun más globales y con una visión transformadora.

5. La enseñanza virtual debe incluir en sus procesos el fin último que se desea; un profesional con mentalidad abierta, con procesos globalizados, con visión internacional, con carácter científico, con habilidades técnicas, con recursos para la adaptación al cambio, con conocimientos para el trabajo en equipo y multicultural.

\section{CONCLUSIONES}

Una vez finalizado el proceso de revisión, es posible realizar las siguientes conclusiones al respecto:

1. La educación universitaria requiere un cambio para poder adaptarse a las necesidades sociales, y este cambio no necesariamente debe ser de paradigmas, sino de formas y metodologías de enseñanza, que se acoplen a nuevos procesos industriales y nuevas visiones globales económicas y sociales. 
2. Las transiciones sociales son cada vez más fuertes, en un mismo año es posible observar diversos cambios en la sociedad que conducen a una diversidad de cambios en los sectores industriales, comerciales, económicos, políticos, y culturales. Estas transformaciones necesarias, forman ciudadanos con habilidades diferentes, con una variedad de exigencias distintas a las de tiempos anteriores, y por ende, la educación no puede continuar con métodos tradicionales.

3. Los métodos tradicionales siguen siendo la principal forma de enseñanza, a pesar de incorporar diversos cambios en las metodologías, sigue siendo el proceso tradicional el que prevalece. De ahí que la transformación aun no es visible ni es palpable.

4. Resulta preocupante que la industria mundial esté adaptando características tecnológicas y científicas que permiten una competitividad globalizada, sin embargo, en Latinoamérica aun no existe una formación profesional para atender a las inquietudes de esa industria. 


\section{REFERENCIAS}

Calizaya, J., Zapata, F., Monzón, G., Alemán, Y., Bellido, R., \& Ceballos, F. (2020). La investigación cuantitativa en las ciencias sociales y algunas aplicaciones de interés científico. Quito, Ecuador: AutanaBooks.

Dunfey, T. (2019). What is Social Change and Why Should We Care? Southern New Hampshire University.

Guichot, V. (2006). HISTORIA DE LA EDUCACIÓN: REFLEXIONES SOBRE SU OBJETO, UBICACIÓN EPISTEMOLÓGICA, DEVENIR HISTÓRICO Y TENDENCIAS ACTUALES. Revista Latinoamericana de Estudios Educativos, 2(1), 11-51.

La importancia de las letras. (2010). La historia de la educación. Recuperado el 27 de 11 de 2021, de http://historiageneraldelaeducacion.blogspot.com/2010/03/historia-de-la-educacion-conclusion.html

Lenski, G. E. (1974). Human Societies: An Introduction to Macrosociology. New York:: McGraw-Hill,. Oficina de información diplomática. (2021). República de Singapur. Recuperado el 23 de noviembre de 2021, de http://www.exteriores.gob.es/documents/fichaspais/singapur_ficha\%20pais.pdf

Pixabay. (s.f.). Recuperado el 22 de noviembre de 2021, de https://pixabay.com/es/

Scafatti, L. (s.f.). Sociedad. Recuperado el 29 de noviembre de 2021, de https://www.mendoza.conicet.gov.ar/portal/enciclopedia/terminos/Sociedad.htm

Ssociólogos. (11 de julio de 2012). La teoría de las estructuras sociales de acumulación. Recuperado el noviembre de 23 de 2021, de https://ssociologos.com/2012/07/11/la-teoria-de-las-estructuras-sociales-deacumulacion/

Westreicher, G. (09 de septiembre de 2020). Sociedad. Recuperado el 23 de noviembre de 2021, de https://economipedia.com/definiciones/sociedad.html

Westreicher, G. (09 de septiembre de 2020). Sociedad. (Economipedia) Recuperado el 29 de noviembre de 2021, de https://economipedia.com/definiciones/sociedad.html

Zambrano, K. (13 de septiembre de 2018). Línea de tiempo de la historia de la educación. Recuperado el 24 de noviembre de 2021, de https://prezi.com/p/oashlaqm_uxn/linea-del-tiempo-historia-de-laeducacion/ 